\title{
Semantically Enriched Services to Understand the Need of Entities
}

\author{
Flávio de Oliveira Silva ${ }^{1,3}$, Alex Dias ${ }^{2}$, Caio César Ferreira ${ }^{3}$, \\ Eduardo De Souza Santos ${ }^{3}$, Fabíola Souza Fernandes Pereira ${ }^{4}$, \\ Isabelle Cecília de Andrade ${ }^{3}$, João Henrique de Souza Pereira ${ }^{1}$, \\ Lásaro Jonas Camargos ${ }^{3}$, Luiz Cláudio Theodoro ${ }^{3}$, \\ Maurício Amaral Gonçalves ${ }^{3}$, Rafael Pasquini ${ }^{3}$, Augusto José Venâncio Neto ${ }^{5}$, \\ Pedro Frosi Rosa ${ }^{3}$, and Sergio Takeo Kofuji ${ }^{1}$ \\ 1 University of São Paulo, Brazil \\ \{flavio,kofuji\}@pad.lsi.usp.br, joaohs@usp.br \\ 2 Federal Institute of Triângulo Mineiro, Brazil \\ alexdias@iftm.edu.br \\ 3 Federal University of Uberlândia, Brazil \\ \{caiocf, isabelle, mauricio\}@algartelecom.com.br, eduardo@doutorado.ufu.br, \\ \{lasaro,pasquini,frosi\}@facom.ufu.br, lclaudio@feelt.ufu.br \\ 4 Algar Telecom, Brazil \\ fabiolas@algartelecom.com.br \\ 5 Federal University of Ceará, Brazil \\ augusto.deti@ufc.br
}

\begin{abstract}
Researchers from all over the world are engaged in the design of a new Internet, and Software-Defined Networking (SDN) is one of the results of this engagement. Net-Ontology uses a SDN approach to bring semantics to the intermediate network layers and make them capable of handling application requirements and adapt their behaviour over time as required. In this paper we present an experimental evaluation of Net-Ontology and a feature comparison against the traditional TCP/IP stack. This paper extends our earlier work towards a Future Internet, showing a viable approach to introduce semantics at network lower layers by contributing to bring richer and efficient services.
\end{abstract}

Keywords: Future Internet, Enrich Services, Network Ontology, SDN, DTS, Workspace.

\section{Introduction}

The evolution of the intermediate network layers have been lagging behind that of the lower and upper layers. The Internet Protocols, specified more than three decades ago, are the likely culprit; the application needs have changed by leaps, while the TCP/IP has only been patched, trying to meet these requirements. Over the last few years, the networking community has strived to correct this phenomenon [1, 3, 4, 21]. 
Researchers from all over the world are engaged in the design of a new Internet, from the ground up. This so called clean slate approach, frees the research from the legacy of the current architecture and fosters innovations 18]. At a future time, when results should be deployed, the research will then be refocused to the transition from the current Internet to the future Internet

One of the results of this effort to create the Future Internet is Software-Defined Networking (SDN) [5, 6]. SDN enables researchers to innovate and experiment new network protocols, naming and addressing schemes, such as the one presented in this paper, which aims at bridging the evolutionary gap between upper, lower, and the intermediate network layers by using a richer semantics [15, 16].

FINLAN (Fast Integration of Network Layers) [9, 13, 14, 19] aims at providing high adaptability through the use of semantic concepts based on ontology, with the elimination of static routing and addressing tied to physical location, resulting in a better and efficient utilization of the network infrastructure.

FINLAN defines two intermediate layers that communicate between each other using OWL (Web Ontology Language), but that clearly differentiate in function: DL-Ontology and Net-Ontology.

The DL-Ontology layer is essentially responsible for data transfer between the Physical layer and the upper layers, handling the semantic communication between the peer entities and bringing a richer capacity to express their requirements. On the other hand, the Net-Ontology layer is accountable for handling service needs, as it is capable of understanding specific requirements from the application and adapting the communication to support them only when required, using DL-Ontology to deal with the semantic communication.

In this chapter we present the Net-Ontology layer, which sits between the DL-Ontology layer and the application. We also present its implementation and a first experimental evaluation. The implementation presented is based on the Title Model[17], our vision regarding future networks.

The remainder of this work is organized as follows: Section 1 describes the Net-Ontology. Section 2 shows the Net-Ontology implementation and Section 3 the experimental results. The conclusions are presented in Section 4.

\section{The Net-Ontology}

The DL-Ontology is the lower layer of the FINLAN stack depicted in Figure [1. and enables the communication using concepts expressed in OWL over the Physical layer.

The Net-Ontology layer is responsible for supporting the service needs of the upper layer and deliver them to the DL-Ontology layer, built according to the FINLAN Ontology. In this approach, the Net-Ontology is able to understand specific requirements of a given application that may arise over communication and provide them.

For example, let us suppose that two persons, $P_{1}$ and $P_{2}$, are chatting over the Internet, using the application FinChat that runs over the FINLAN stack. In a certain moment, they want to start a secret conversation. To FinChat meet 
this need, the only thing it has to do is to inform the Net-Ontology layer that from now on the chat is to be confidential. The Net-Ontology layer is able to understand this need and act accordingly modifying all packets exchanged from that moment.

The Net-Ontology consists, basically, of two main modules: requirement analysis and requirement manager, as depicted in Figure 1 .

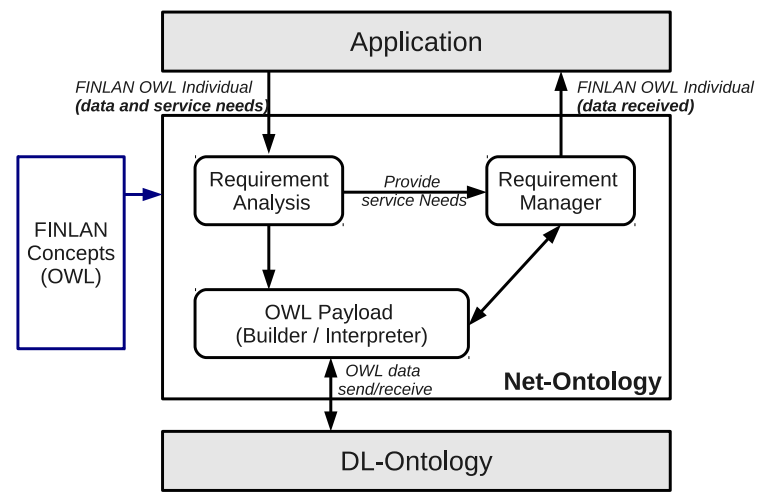

Fig. 1. Net-Ontology modules

The requirement analysis module (RAM) is responsible for handling the application requests regarding communication requirements. To accomplish this, RAM uses the Leśniewski's logic as proposed in [8]. The purpose is to manage the services requirements over time. This module recognizes what technological features are necessary to satisfy a given requirement, in a given moment, combining them in logical formulas.

As an example, let us suppose that a service $S 1$, in a moment $t_{1}$, may need to establish communication with the service $S 2$, with a specific requirement. The RAM will verify that this upper layer requirement can be provided by the technological requirements $R 1$ and $R 2$. In another moment $t_{2}, S 1$ wishes to improve security, using confidentiality in the conversation. For so, it is necessary the technological requirement $R 3$. These scenarios will be interpreted by the analysis module and represented by the following axioms:

$$
\begin{gathered}
S 1 S 2 t_{1} \rightarrow R 1 \wedge R 2 \\
S 1 S 2 t_{2} \rightarrow(R 1 \wedge R 2) \wedge R 3
\end{gathered}
$$

The requirement manager module (RMM) takes the rules requirements and transform them into FINLAN ontology fragments. Besides that, this module is able to interpret and deploy the algorithms correlated with each requirement of the ontology in the network stack. 
Taking the aforementioned example, in the moment $t_{1}$, RMM receives the requirements $R 1$ and $R 2$ from RAM. It will then use the FINLAN Ontology, and add to the packages an OWL fragment, representing that $P_{1}$ has requirements $R_{1}$ and $R_{2}$ in FinChat.

From now on, the packages will be transmitted containing new information. When the FinChat of $P_{2}$ receives an OWL package, meaning that $R 1$ and $R 2$ are required, the RMM will be able to understand and make use of the necessary algorithms. The intelligence for the network to understand and implement the applications needs is the main responsibility of the requirement manager module.

The requirements, manipulated by RAM, are stored at the Domain Title Service (DTS), which consists of a distributed system over the network elements responsible for maintaining the entities available in a domain and their communication requirements over time. It plays an important role at central aspects of networking like naming and addressing, and has the ability to share the context among communicating entities. This sharing is provided by the workspace.

The workspace is a logical bus which contains network elements required to support the communication of the entities. The workspace is created by an entity wiling to communicate with a specific purpose and thus defines its requirements and capabilities. A new entity can be joined to an existing workspace and, in such event, the logical bus can be adapted to handle its communication.

All entities that shares a workspace takes part in the same communication. The data is sent once by a source to the workspace and is received by all the others, thus making an efficient use of the physical layer.

In the next subsection it will be presented a complete case of how the NetOntology modules interact with the others FINLAN layers and the DTS.

\subsection{FINLAN Semantic Communication}

The communication between the FINLAN layers occurs in a semantic way, by using OWL. Below, it is presented an example to illustrate how this communication happens.

Let us suppose a scenario where John and Paul are chatting using the application FinChat that runs over the FINLAN layers, through the workspace WKS.1. In a first moment $t_{1}$, they are just talking about irrelevant issues and are not concerned about any additional feature that FinChat can offer to them. So, the packages travelling in the network are very simple, and the Net-Ontology has not introduced any new requirement at the communication, in this case, only the DL-Ontology handles their communication. A code snippet example can be:

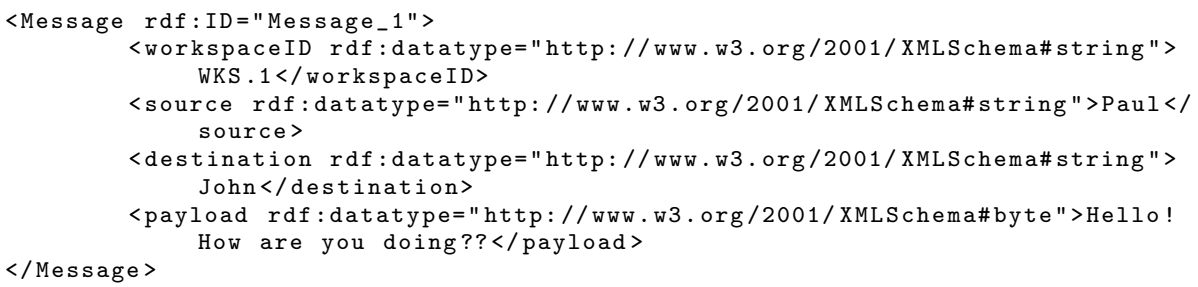


After some time, at instant $t_{2}$, John starts an important subject, and selects the feature delivery guarantee of FinChat. This means that from now on, FinChat requires delivery guarantee to the network. The Figure 2 shows the messages flow that will be sent and received between the FinChat entities and the DTS, to attend this request.

With a new requirement, the Net-Ontology layer is triggered, and the requirement analysis module checks that it is necessary the technological requirement of a delivery guarantee algorithm. John's FinChat, then, sends the following control message to DTS:

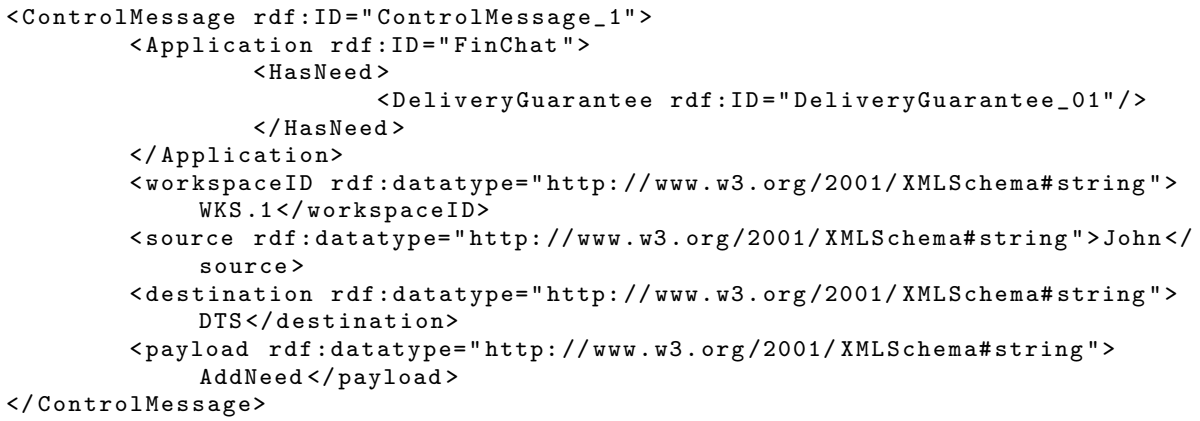

After registering John's need, the DTS will send him a confirmation message:
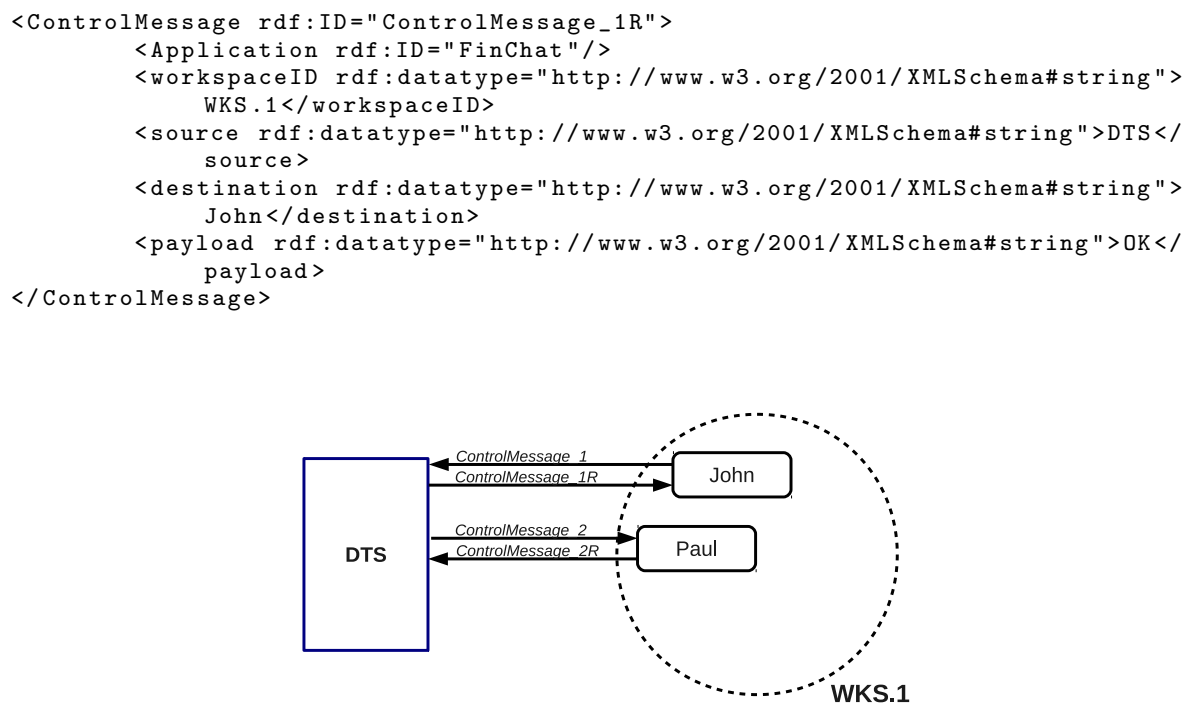

Fig. 2. Message flow example for a new requirement

At the same time, DTS will also send to Paul, who is in the same workspace as John, a control message, asking if the need requested is supported: 


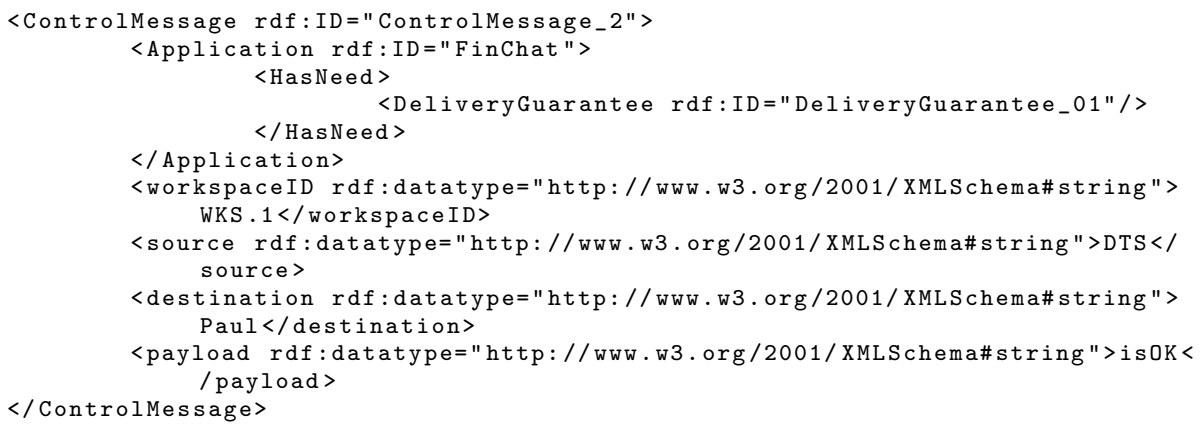

If Paul's FinChat can supply the delivery guarantee feature, the response below is sent to DTS and it is established a communication with support to delivery guarantee:

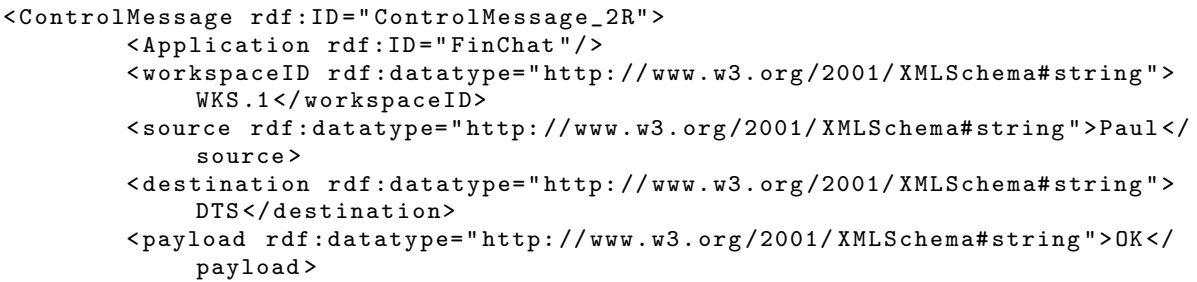

In case of Paul's FinChat with no support for the delivery guarantee, this feature will not be present in the communication between both applications.

Notice that through the Net-Ontology, FINLAN is able to register the services needs into the DTS. From now on, it can manage what is the best way to deliver FINLAN packages.

If a third person, Ringo, wants to join the conversation, Ringo's FinChat will handshake with DTS to check if it has support to DeliveryGuarantee_01. This scenario is illustrated in Figure 3 .
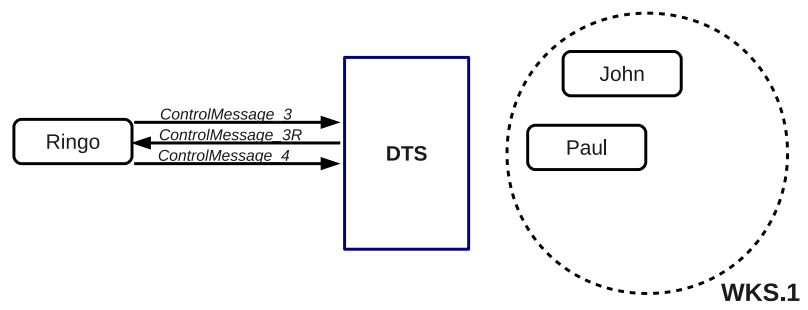

Fig. 3. Message flow example for a Join into Workspace WKS.1

The following messages are exchanged and Ringo joins the workspace WKS.1. After the joining and, hence, sharing of the workspace, Ringo's FinChat and all the other entities will receive the same data messages without the need of multiple data flows. 


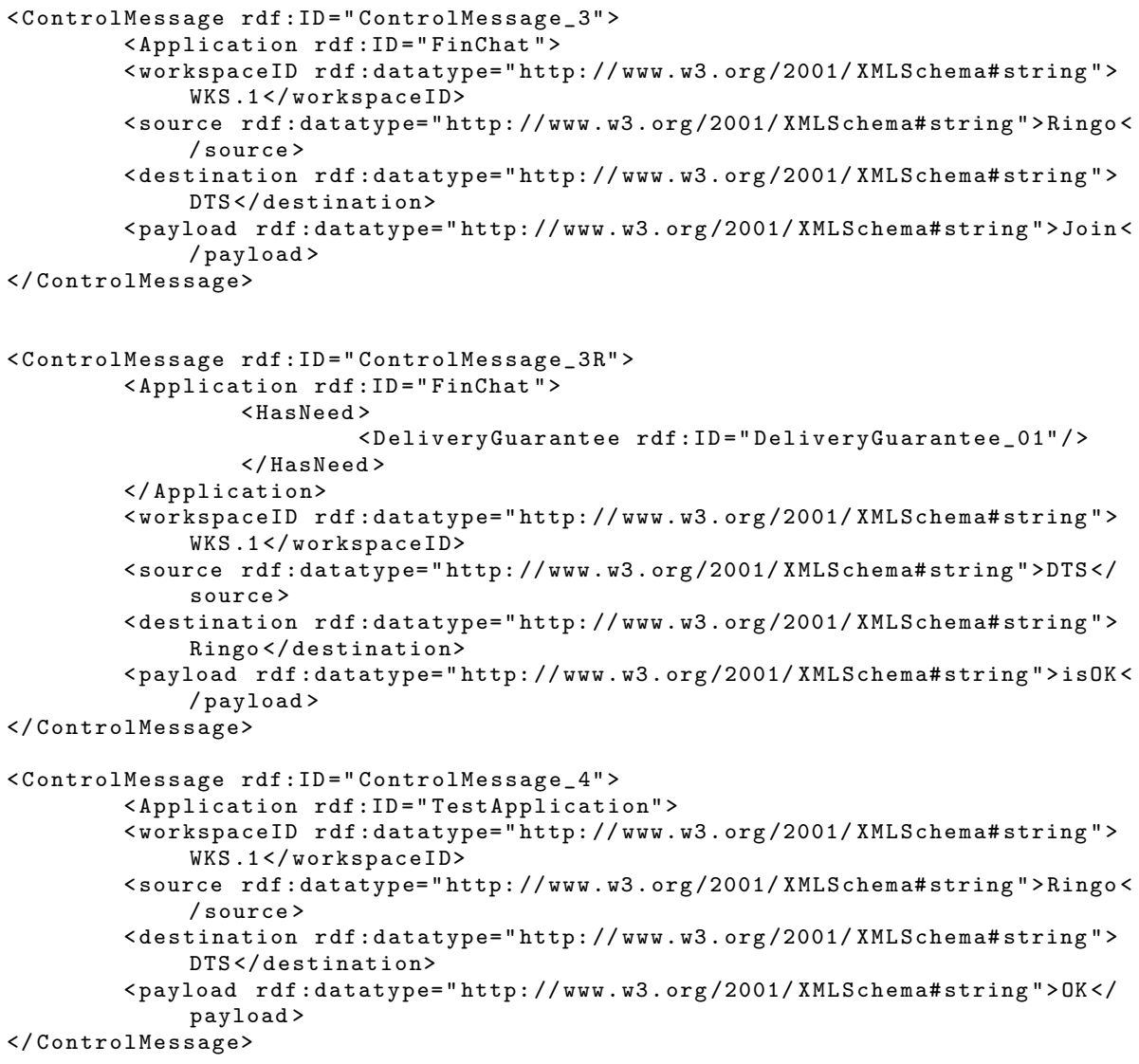

It is also important to mention that after the exemplified handshakes between DTS and FinChat entities, the requirement manager module is responsible for guaranteeing that the exchanged packages during the chat will have the necessary information, implementing the algorithm DeliveryGuarantee_01. For example, differently from the Message_1 structure, the messages must have an identification field, through which the control of lost packages is made.

\section{Implementation}

Our FINLAN stack implementation consists of a Java library that uses communication interfaces through Raw Sockets. The linking between Java and $\mathrm{C}$ portions of the code was done in Java Native Interface (JNI) [16, 19], as depicted in Figure 4 .

It is observed that the application App.java should use the API available in the library Finlan.jar to establish communication. In this way, when an application sends a packet, it communicates with the Net-Ontology sending its characteristics. According to these characteristics, the Requirement Module Analisys determines, through an inference engine, the application needs and proceeds 


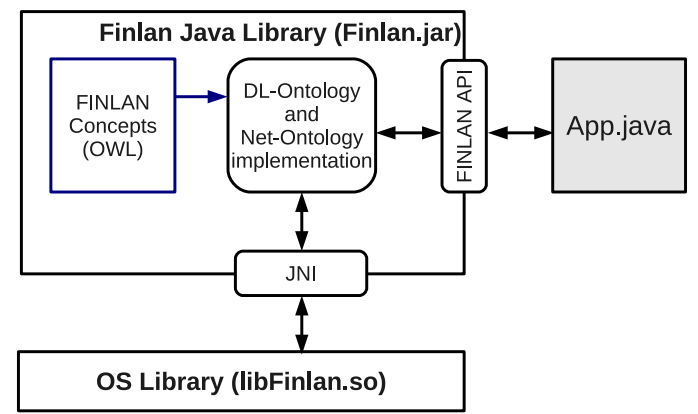

Fig. 4. FINLAN Modules Implementation

with the delivery of these. After the completion of the relevant operations, NetOntology sends the primitive to the DL-Ontology which, in turn, takes care of sending the packet through the JNI interface to the libFinlan.so library.

\section{Experimental Results}

To validate the implementation of this work, it was performed experiments comparing FINLAN with the TCP/IP Architecture protocols. The goal of these experiments was to show the behavior of the ontology use in a file transfer operation with the delivery guarantee need activated, illustrating the use of the Net-Ontology layer in FINLAN.

The TCP/IP protocols, by definition, already implements the delivery guarantee feature, when the TCP transport protocol is used. To FINLAN support this need, this work implemented it in the Net-Ontology layer, using the algorithm described in [14]. This algorithm is a mechanism to ensure that all packages sent are received and works as follows: when the need delivery guarantee is activated by the application layer, all packages, sent from this moment contains a new field representing the number of the package.

In parallel, there is a mechanism of confirmation requests and responses messages: the source host informs the packages already sent, requesting the lost ones. The destination, on the other hand, answers which packages it did not receive. This change of confirmation messages is orchestrated by the RTT variable, proposed in [7], which consists of the best estimate (for that moment) for the send and receive time up of the packets destination.

\subsection{Network Traffic Evaluation}

The experiments were performed over the following environment: hosts with 4GB of RAM, CPU Intel ${ }^{\circledR}$ Core $^{\mathrm{TM}_{2}}$ DUO @ $2.10 \mathrm{GHz}$, running Linux operational system with kernel 2.6.41.10-3.fc15.x86_64. The files transfered have size of 1, 5, $10,15,20,25,30,35,40,45$ and 50MB. The RTT variable was set to a fixed 


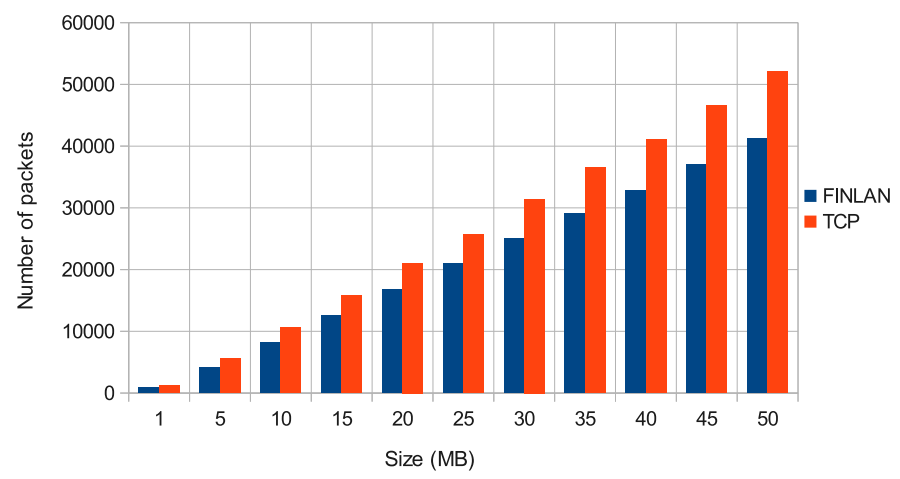

(a) Number of packets

\begin{tabular}{|c|c|c|c|}
\hline Size & FINLAN & TCP & Reduction (\%) \\
\hline $1 \mathrm{MB}$ & 847 & 1168 & $37,9 \%$ \\
\hline $5 \mathrm{MB}$ & 4174 & 5594 & $34,0 \%$ \\
\hline $10 \mathrm{MB}$ & 8140 & 10631 & $30,6 \%$ \\
\hline $15 \mathrm{MB}$ & 12566 & 15733 & $25,2 \%$ \\
\hline $20 \mathrm{MB}$ & 16722 & 21060 & $25,9 \%$ \\
\hline $25 \mathrm{MB}$ & 20920 & 25277 & $25,6 \%$ \\
\hline $30 \mathrm{MB}$ & 25017 & 31423 & $25,6 \%$ \\
\hline $35 \mathrm{MB}$ & 29089 & 36495 & $25,5 \%$ \\
\hline $40 \mathrm{MB}$ & 32786 & 41143 & $25,5 \%$ \\
\hline $45 \mathrm{MB}$ & 37070 & 46585 & $25,7 \%$ \\
\hline $50 \mathrm{MB}$ & 41207 & 52067 & $26,4 \%$ \\
\hline
\end{tabular}

(b) Percentual reduction

Fig. 5. FINLAN and TCP traffic comparison

value of 1 second. Figure [5] shows the results, comparing the number of packets transmitted in both: FINLAN and TCP.

It is possible to observe that in the scenarios of this experimentation, FINLAN had a smaller number of transmitted packets. In the transfer operation of $10 \mathrm{MB}$, for example, FINLAN transmitted 8140 packets, while the TCP transmitted 10631 (one difference of 30.6 percent).

This is due to the delivery guarantee algorithm implemented in FINLAN that sends confirmation messages in intervals of the RTT, informing only the lost ones, in a period, to be re-transmitted, while TCP transmitted several number of ACK packages. This confirms that the network traffic packets is decreased using the delivery guarantee algorithm implemented over a stack that semantically understands the concepts and adapts the messages from this understanding.

To illustrate the primitives in these experiments, Figure 6] shows snapshots from the Wireshark of two packets captured during the transmission of the $50 \mathrm{MB}$ file. The first one, in Figure 6(a) is the confirmation request of the source entity, called "fabiola", informing that the range of packages from 133 to 367 was sent. The Figure 6(b) represents the response, confirming the lost packages, through 


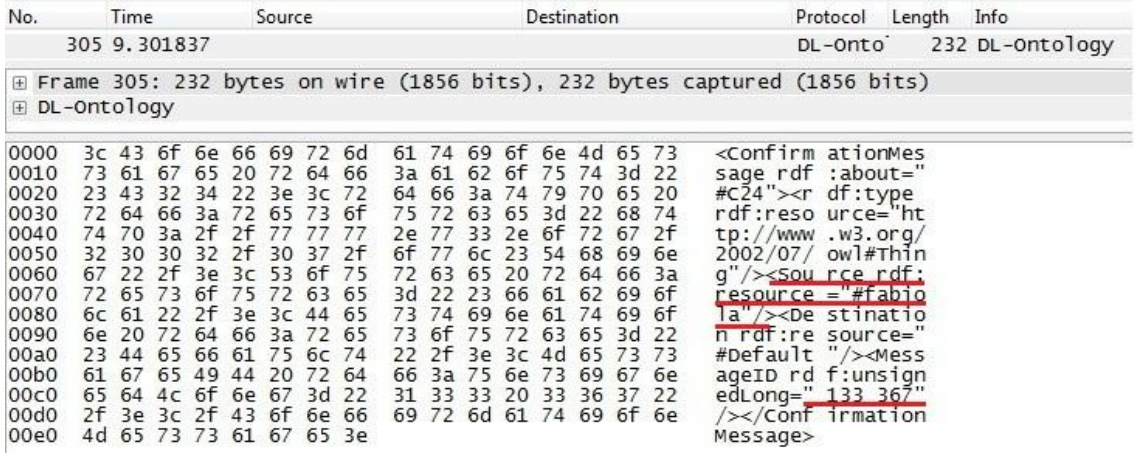

(a) Confirmation request

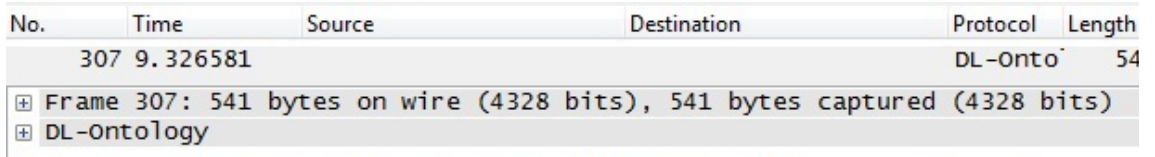

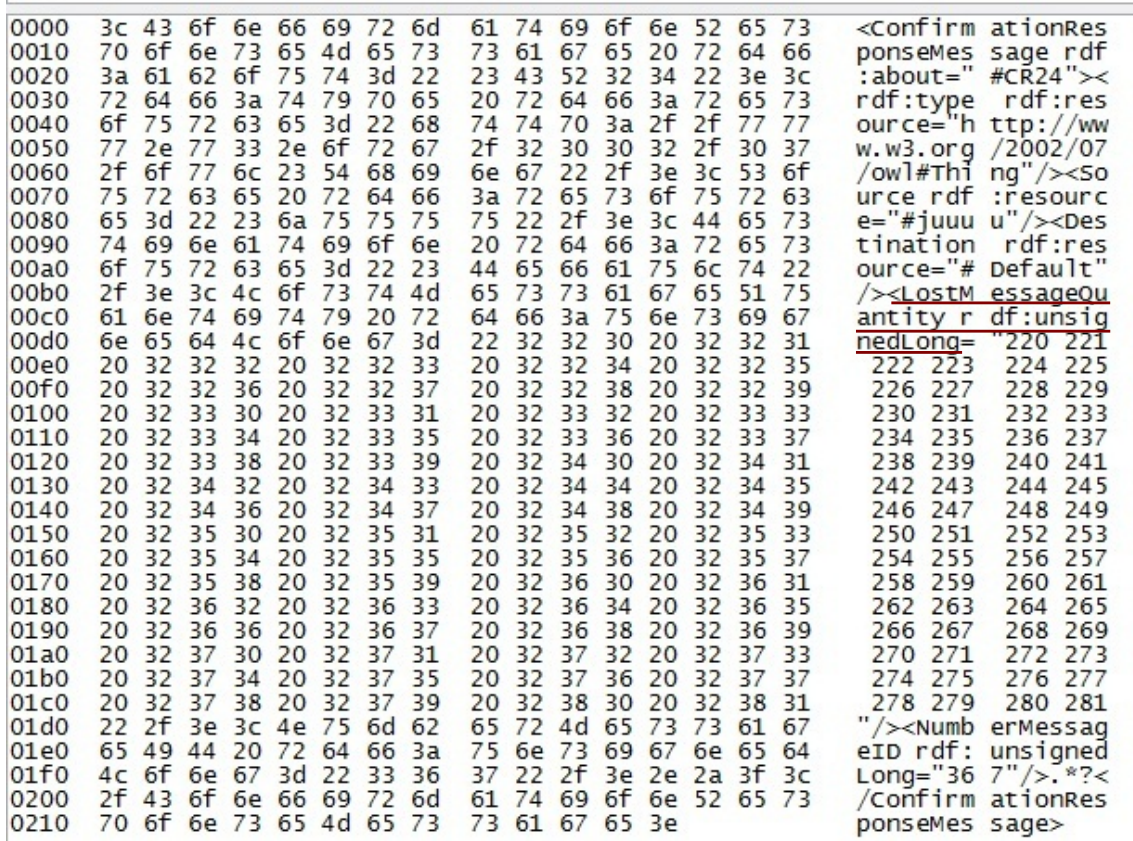

(b) Confirmation response

Fig. 6. Snapshots of FINLAN confirmation messages

the field LostMessageQuantity. According to this capture, the packages from 220 to 281 were lost and only them were re-transmitted. 


\section{Conclusions}

This work presented the Net-Ontology Layer, experimental results of its implementation and how it is possible to use ontology at the intermediate networks layers to understand and support different entities needs.

The results of using ontology to support the delivery guarantee need demonstrate an optimization of more than 30 percent of the packets sent in a file transfer, compared with the traditional TCP/IP protocols usage.

By the Net-Ontology use, it was demonstrated the possibility to substitute the traditional TCP/IP protocols used at the transport and network layers. This brings more semantic power for the Future Internet networks, as the network intermediate layers become able to better understand the entities needs.

Future Internet is being constructed with worldwide collaboration and is based on research and experimentation. Our previous work showed [17, 19] how FINLAN approach and the Title Model Ontology can work together with different efforts regarding the future, while the work presented details on how these proposals can come true.

As future works, it is expected to experiment the Net-Ontology implementation in different testbeds, such as OFELIA [11] and FIBRE (Future Internet testbeds/experimentation between BRazil and Europe) [2, 20]. In complement, it will be finished the actual working in progress to the experimentation using OpenFlow [10]. Also, experimental tests using workspaces for multicast aggregation [12] are being executed at OFELIA testbed.

The research and experimentation results show that we are facing a viable approach to introduce semantics at network lower layers, by contributing to bring richer and efficient services.

Acknowledgment. This work received contributions from the FINLAN project and other researchers from the MEHAR program. The authors would like to thank all FINLAN and MEHAR members for the extensive discussion and collaboration to the Future Internet perspectives.

Open Access. This article is distributed under the terms of the Creative Commons Attribution Noncommercial License which permits any noncommercial use, distribution, and reproduction in any medium, provided the original author(s) and source are credited.

\section{References}

[1] Clayman, S., Galis, A., Chapman, C., Toffetti, G., Rodero-Merino, L., Vaquero, L.M., et al.: Monitoring Service Clouds in the Future Internet. Towards the Future Internet - Emerging Trends from European Research, p. 115 (2010)

[2] FIBRE: FIBRE Project (January 2012), http://www.fibre-ict.eu/

[3] FIRE: FIRE White Paper (August 2009), http://www.ict-fireworks.eu/ fileadmin/documents/FIRE_White_Paper_2009_v3.1.pdf 
[4] Galis, A., Denazis, S., Bassi, A., Giacomin, P., Berl, A., Fischer, A., et al.: Management Architecture and Systems for Future Internet. Towards the Future Internet - A European Research Perspective, p. 112 (2009)

[5] Greenberg, A., Hjalmtysson, G., Maltz, D.A., Myers, A., Rexford, J., Xie, G., Yan, H., Zhan, J., Zhang, H.: A clean slate 4d approach to network control and management. SIGCOMM Comput. Commun. Rev. 35, 41-54 (2005), http://doi.acm.org/10.1145/1096536.1096541

[6] Greene, K.: TR10: Software-Defined Networking. MIT - Technology Review (2009)

[7] Jacobson, V.: Congestion Avoidance and Control. SIGCOMM Communications Architectures and Protocols, USA 88, 314-329 (1988)

[8] Leśniewski, S.: Comptes rendus des séances de la Société des Sciences et des Lettres de Varsovie. Class III, pp. 111-132 (1930)

[9] Malva, G.R., Dias, E.C., Oliveira, B.C., Pereira, J.H.S., Kofuji, S.T., Rosa, P.F.: Implementação do Protocolo FINLAN. In: 8th International Information and Telecommunication Technologies Symposium (2009)

[10] McKeown, N., Anderson, T., Balakrishnan, H., Parulkar, G., Peterson, L., Rexford, J., Shenker, S., Turner, J.: OpenFlow: Enabling Innovation in Campus Networks. SIGCOMM Comput. Commun. Rev. 38, 69-74 (2008)

[11] OFELIA: OFELIA Project (January 2012), http://www.fp7-ofelia.eu/

[12] de Oliveira Silva, F.: Experimenting domain title service to meet mobility and multicast aggregation by using openflow. In: MyFIRE Workshop (September 2011)

[13] Pereira, F.S.F., Santos, E.S., Pereira, J.H.S., Rosa, P.F., Kofuji, S.T.: Proposal for Hybrid Communication in Local Networks. In: 8th International Information and Telecommunication Technologies Symposium (2009)

[14] Pereira, F.S.F., Santos, E.S., Pereira, J.H.S., Rosa, P.F., Kofuji, S.T.: FINLAN Packet Delivery Proposal in a Next Generation Internet. In: IEEE International Conference on Networking and Services, p. 32 (2010)

[15] Pereira, J.H.S., Pereira, F.S.F., Santos, E.S., Rosa, P.F., Kofuji, S.T.: Horizontal Address by Title in the Internet Architecture. In: 8th International Information and Telecommunication Technologies Symposium (2009)

[16] Pereira, J.H.S., Santos, E.S., Pereira, F.S.F., Rosa, P.F., Kofuji, S.T.: Layers Optimization Proposal in a Post-IP Network. International Journal on Advances in Networks and Services (2011)

[17] Pereira, J.H.S., Silva, F.O., Filho, E.L., Kofuji, S.T., Rosa, P.: The Title Model Ontology for Future Internet Networks. In: Domingue, J., et al. (eds.) FIA 2011. LNCS, vol. 6656, pp. 103-114. Springer, Heidelberg (2011)

[18] Roberts, J.: The clean-slate approach to future internet design: a survey of research initiatives. annals of telecommunications - annales des télécommunications 64(5-6), 271-276 (2009), http://www.springerlink.com/content/e240776641607136/

[19] Santos, E., Pereira, F., Pereira, J.H.: Meeting Services and Networks in the Future Internet. In: Domingue, J., et al. (eds.) FIA 2011. LNCS, vol. 6656, pp. 339-350. Springer, Heidelberg (2011)

[20] Stanton, M.: FIBRE-EU and FIBRE-BR (October 2011), http://www.futureinternet.eu/fileadmin/documents/poznan_documents/Session2_3_Inter national_coop/2-3-stanton.pdf

[21] Tselentis, G., Domingue, J., Galis, A., Gavras, G., Hausheer, D., Krco, S., Lotz, V., Zahariadis, T.: Towards the future internet a European research perspective. IOS Press, Amsterdam (2009) 